\title{
GENERIC RANK-TWO PERTURBATIONS OF STRUCTURED REGULAR MATRIX PENCILS
}

\author{
LEONHARD BATZKE
}

Abstract. The generic spectral behavior of classes of structured regular matrix pencils is examined under structure-preserving rank-2 perturbations, i.e., perturbations of normal rank two. For $T$-alternating, palindromic, and skew-symmetric matrix pencils we observe the following effects at each eigenvalue $\lambda$ under a generic, structure-preserving rank-2 perturbation: 1) The largest two Jordan blocks at $\lambda$ are destroyed. 2) If hereby the eigenvalue pairing imposed by the structure is violated, also the largest remaining Jordan block at $\lambda$ will grow in size by one. 3 ) If $\lambda$ is a single (double) eigenvalue of the perturbating pencil, one (two) new Jordan blocks of size one will be created at $\lambda$.

Mathematics subject classification (2010): 15A18, 15A21, 15A22, 15B57, 47A55.

Keywords and phrases: Matrix pencil, alternating matrix pencil, palindromic matrix pencil, skewsymmetric matrix pencil, perturbation theory, rank two perturbation, generic perturbation.

\section{REFERENCES}

[1] L. BATZKe, Generic rank-one perturbations of structured regular matrix pencils, Linear Algebra Appl., 458: 638-670, 2014.

[2] F. DE TERÁn AND F. DOPICO, A note on generic Kronecker orbits of matrix pencils with fixed rank, SIAM J. Matrix Anal. Appl., 30: 491-496, 2008.

[3] F. De Terán, F. DOPICO And J. Moro, Low rank perturbation of Weierstrass structure, SIAM J. Matrix Anal. Appl., 30: 538-547, 2008.

[4] F. DE TERÁN AND F. DOPICO, Generic change of the partial multiplicities of regular matrix pencils under low-rank perturbations, Submitted for publication, 2015.

[5] A. Edelmann, E. Elmroth And B. KÅgström, A geometric approach to perturbation theory of matrices and matrix pencils. Part I: Versal deformations, SIAM J. Matrix Anal. Appl., 18: 653-692, 1997.

[6] A. Edelmann, E. Elmroth And B. KÅgStröm, A geometric approach to perturbation theory of matrices and matrix pencils. Part II: A stratification-enhanced staircase algorithm, SIAM J. Matrix Anal. Appl., 20: 667-699, 1999.

[7] J. H. Fourie, G. J. Groenewald, D. B. Janse van Rensburg And A. C. M. Ran, Rank one perturbations of H-positive real matrices, Linear Algebra Appl., 439: 653-674, 2013.

[8] L. Hörmander And A. Melin, A remark on perturbations of compact operators, Math. Scand., 167: 113-118, 1994.

[9] P. LANCASTER AND L. RoDMAN, Canonical forms for symmetric/skew-symmetric real matrix pairs under strict equivalence and congruence, Linear Algebra Appl., 406: 1-76, 2005.

[10] D. S. Mackey, N. Mackey, C. Mehl and V. Mehrmann, Structured polynomial eigenvalue problems: Good vibrations from good linearizations, SIAM J. Matrix Anal. Appl., 28 (4): 1029-1051, 2006.

[11] D. S. Mackey, N. Mackey, C. Mehl and V. Mehrmann, Möbius transformations of matrix polynomials, Technical Report No. 1051, DFG Research Center Matheon, Berlin, 2014.

[12] C. Mehl, V. Mehrmann, A. C. M. Ran And L. Rodman, Eigenvalue perturbation theory of classes of structured matrices under generic structured rank one perturbations, Linear Algebra Appl., 435: 687-716, 2011. 
[13] C. Mehl, V. Mehrmann, A. C. M. Ran and L. Rodman. Perturbation theory of selfadjoint matrices and sign characteristics under generic structured rank one perturbations, Linear Algebra Appl., 436: 4027-4042, 2012.

[14] C. Mehl, V. Mehrmann, A. C. M. Ran And L. Rodman, Jordan forms of real and complex matrices under rank one perturbations, Oper. Matrices, 7: 381-398, 2013.

[15] C. Mehl, V. Mehrmann, A. C. M. Ran and L. Rodman, Eigenvalue perturbation theory of symplectic, orthogonal, and unitary matrices under generic structured rank one perturbations, BIT, 54: 219-255, 2014.

[16] J. Moro AND F. DopiCo, Low rank perturbation of Jordan structure, SIAM J. Matrix Anal. Appl., 25: 495-506, 2003.

[17] S. V. SAVChENKO, Typical changes in spectral properties under perturbations by a rank-one operator, Mat. Zametki, 74: 590-602, 2003. (Russian). Translation in Mathematical Notes. 74: 557-568, 2003.

[18] S. V. SAVChEnKo, On the change in the spectral properties of a matrix under a perturbation of sufficiently low rank, Funkts. Anal. Prilozh., 38: 85-88, 2004. (Russian). Translation in Funct. Anal. Appl. 38: 69-71, 2004.

[19] S. V. SAVChEnKo, Laurent expansion for the determinant of the matrix of scalar resolvents, Mathematicheskii Sbornik, 196: 121-144, 2005. (Russian). Translation in Sbornik: Mathematics. 196: 743-764, 2005.

[20] C. SChröDER, Palindromic and Even Eigenvalue Problems - Analysis and Numerical Methods, $\mathrm{PhD}$ thesis, Technical University Berlin, Germany, 2008.

[21] R. C. Thompson, Pencils of Complex and Real Symmetric and Skew Matrices, Linear Algebra Appl., 147: 323-371, 1991.

[22] F. TisSEUR AND I. ZABALla, Finite and infinite elementary divisors of matrix polynomials: a global approach, MIMS EPrint 2012.78, Manchester Institute for Mathematical Sciences, The University of Manchester, UK, 2012. 